\title{
STOCHASTICITY IN STAR CLUSTERS: REDUCED RANDOM SAMPLING METHOD
}

\author{
P. de Meulenaer ${ }^{1,2}$, D. Narbutis ${ }^{1}$, T. Mineikis ${ }^{1,2}$ and V. Vansevičius ${ }^{1,2}$ \\ 1 Center for Physical Sciences and Technology, \\ Savanoriu 231, Vilnius LT-02300,Lithuania; philippe.demeulenaer@ff.stud.vu.lt \\ 2 Vilnius University Observatory, Čiurlionio 29, Vilnius LT-03100, Lithuania
}

Received: 2014 August 18; accepted: 2014 October 10

\begin{abstract}
This paper aims to contribute to the debate taking place nowadays on the two extreme schemes of sampling the stellar masses within star clusters, known as Optimal Sampling and Random Sampling. We propose a new method for sampling of stellar masses in star clusters which allows a continuous transition between the Optimal Sampling and the Random Sampling. We use a sample of young star clusters from the literature to calibrate the amount of stochasticity generated by the proposed method.
\end{abstract}

Key words: galaxies: star clusters: general

\section{INTRODUCTION}

The aim of this paper is to tackle the problem of the influence of stellar mass sampling in star clusters on the stochasticity of their integrated colors. Indeed, it is yet undefined how the stellar masses should be sampled from the initial mass function (IMF). On one hand, several studies (e.g., Santos \& Frogel 1997; Deveikis et al. 2008; Fouesneau \& Lancon 2010) implement a random stellar mass sampling from the IMF. In this view, the IMF is seen as a probability density function, according to which stellar masses are randomly drawn. This is referred to as Random Sampling (RS). On the other hand, other studies (e.g., Weidner et al. 2010, 2013; Kroupa et al. 2013) propose the idea that stellar masses could be reproduced by Optimal Sampling (OS) of the IMF. According to the latter view, the mass of the most massive star is exactly determined by the mass of the zero-age star cluster (Kroupa \& Weidner 2003; Pflamm-Altenburg et al. 2007; Weidner et al. 2010). Then, the mass of the second most massive star is related to the mass of the most massive one, the mass of the third massive star to the mass of the second, and so on recursively until all stellar masses are generated (see Equations 9-11 of Kroupa et al. 2013 for details of the OS scheme). Hence, for a given star cluster, there is a deterministic link between the masses of its stars and the cluster's mass. This view is supported by observations of the most massive stars of very young clusters (see compilation of such observations in Weidner et al. 2013), which show that there exists a relationship between the mass of the most massive stars and the mass of their host clusters.

In this paper, we present a new sampling method, hereinafter referred to as Reduced Random Sampling, which can be considered as a continuous transition between the two extremes, RS and OS. The concept of the method is to allow a two-parameter variation and thus to tune the extent of stochasticity that can be decreased to zero-stochasticity (i.e., 
OS) or increased up to full stochasticity (i.e., RS). Then, the best value of the parameter guiding the extent of stochasticity is constrained, making use of the compilation of young star clusters by Weidner et al. (2013).

\section{REDUCED RANDOM SAMPLING METHOD}

Recently, Popescu \& Hanson (2013) presented a sampling method based on OS. In their method, the mass bins are defined around each stellar mass derived by the Kroupa et al. (2013) OS scheme so that the stars are resampled in these bins, introducing a small stochasticity in the mass function. However, one could argue that, in this process, the amount of stochasticity is not constrained by observations.

Here we propose a new sampling method, Reduced Random Sampling (RRS), schemed in Fig. 1, which can be considered as a continuous transition between OS and RS. The four main steps in this method are the following:

- Generate stellar masses using OS for a model cluster of a given mass.

- Define bins around the masses of the OS generated stars.

- Expand or shrink the bin widths by a defined value of the expansion factor $P$. For the bins defined in step $2, P=1$. When the bin widths are expanded, $P>1$, and, when the bin widths are narrowed, $P<1$.

- Resample the mass of the star within the modified bin defined around it.

The effect of the expansion factor $P$, controlling the width of the bins, is illustrated in Fig. 1 ( $P$ is indicated at the top of the upper panels). If we shrink the size of the mass resampling bins $(P \rightarrow 0)$, as sketched in the first column of the panels, no stochasticity is produced; it is the OS case. On the contrary, when we expand the bins twice $(P=2$, second column of the panels), we generate stellar masses which are close to those reproduced by OS, i.e., with small stochasticity in the mass function. If we expand the mass resampling bins more, as sketched in the third column of the panels $(P=6)$, we allow the stellar masses to be resampled further from the masses given by OS, what results in enhanced stochasticity. By expanding the mass resampling bins very widely, the method then reaches RS (last column of the panels in Fig. 1).

In Fig. 1, the impact of shrinkage or expansion of the mass resampling bin can be seen on the scatter of the masses of the most massive stars plotted versus the mass of the host star cluster (third row of the panels) and on the distribution of the integrated colors of these star clusters (bottom row of the panels). As the figure shows, the expansion of the bin increases stochasticity in the integrated photometry of clusters.

In step 1, stellar masses are first sampled optimally by using the method of Kroupa et al. (2013). Here we used the OS algorithm from Pflamm-Altenburg \& Kroupa (2006) ${ }^{1}$. In the OS method, any mass of the most massive star, $m_{\max }$, of a given host cluster of mass $M$ follows exactly a theoretical relation shown in Fig. 2 by the long-dashed cyan line. In this paper, however, we modified the OS scheme so that the mass $m_{\max }$ of the most massive star of a given cluster of mass $M$ follows exactly the empirical relation of Weidner et al. (2013), shown by the green solid line in Figs. 1 and 2, as it represents the observations better (see Fig. 2).

In step 2, we define a bin around each OS star position. The boundaries of the bin are placed at half distance in mass space between the star and its lower- and higher-mass neighbors. Such a bin width, $w_{\mathrm{OS}}$, corresponds to the case when the expansion factor is $P=1$.

\footnotetext{
1 “optimal_sampling”: http://www.astro.uni-bonn.de/en/download/software/
} 

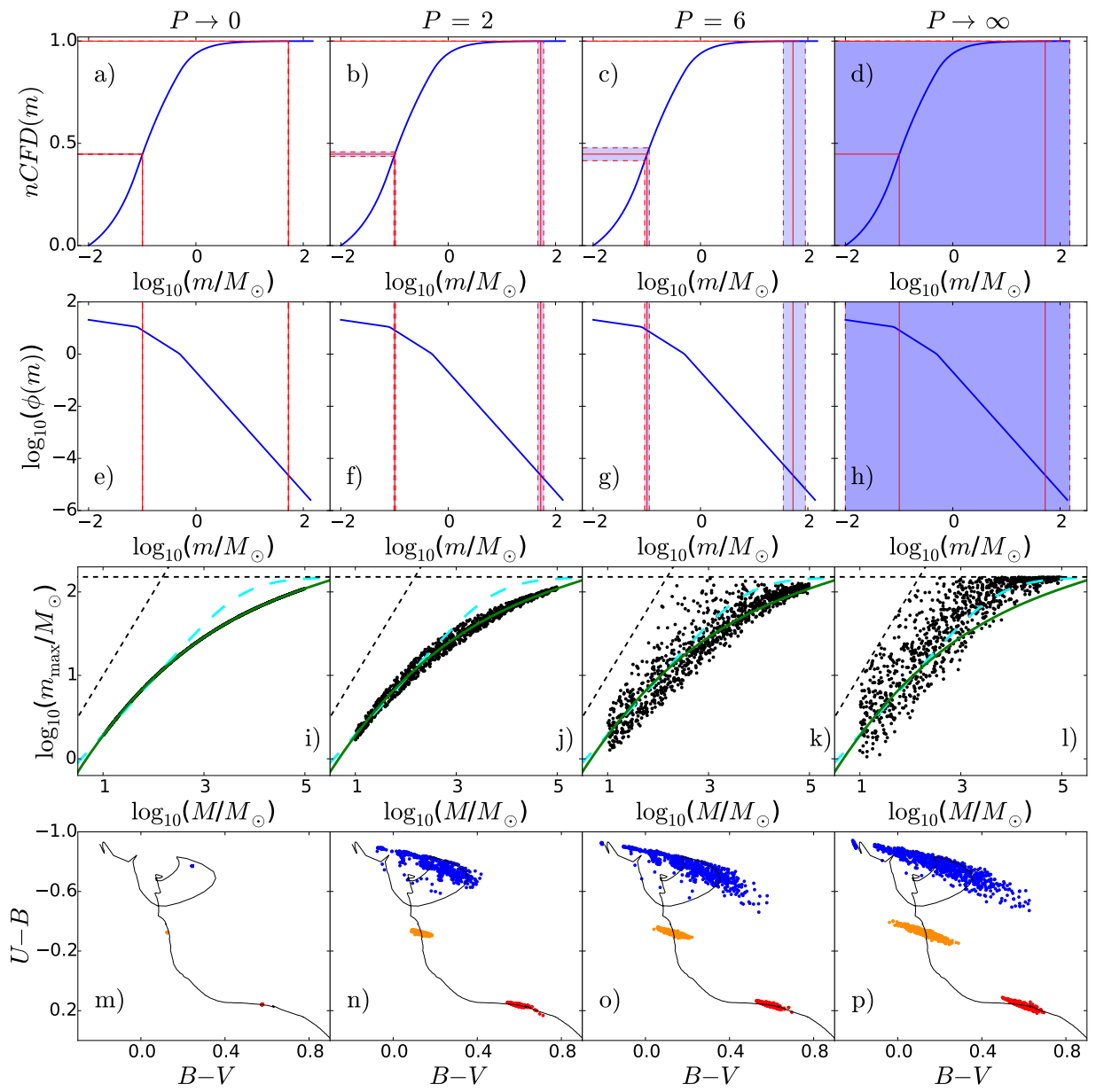

Fig. 1. Scheme of the RRS method, allowing a continuous transition between OS and RS. The columns of the panels represent the results obtained with different values of the expansion factor $P$, indicated on the top. The upper two rows of the panels show the cumulative distribution function and the IMF, respectively. These panels demonstrate the transition between OS and RS, schemed for two stars, one in the low-mass regime and another in the high-mass regime of the IMF. For each star generated by OS, a bin is defined around its mass. For the purpose of illustration, the bin in the low-mass regime is enlarged by a factor of 200 on a linear scale. In these mass bins, the stellar masses are resampled. The third row of the panels shows the relation between the mass of the most massive stars, $m_{\max }$, and the mass of their host clusters, $M$, compared to the Weidner et al. (2013; green solid line) and Kroupa et al. (2013; cyan long-dashed line) relations. The oblique black dashed line is a one-to-one relation between $m_{\max }$ and $M$, and the horizontal black dashed line is the physical upper stellar mass limit, $150 M_{\odot}$. The bottom row of the panels demonstrates the impact of the increase of stochasticity in the cluster stellar mass sampling on the color distributions of these model clusters. The model clusters shown are for the mass $\log _{10}\left(M / M_{\odot}\right)=4$ and ages of $\log _{10}(t / \mathrm{yr})=7,8,9$ (blue, orange, red).

If we randomly generate stars in each of the bins defined, the mean of the generated mass in each bin will not necessarily be equal to the mass of the OS star in the bin. However, this should be the case which allows to ensure that the mass function of the 


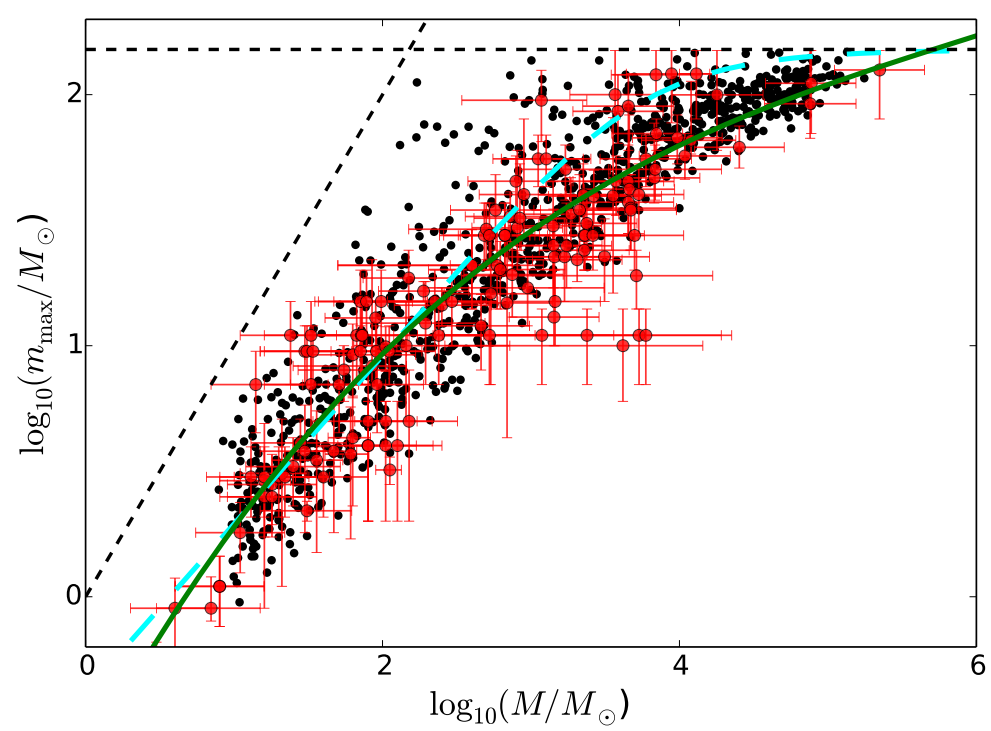

Fig. 2. Sample of the masses of the most massive stars vs. the masses of their host clusters from Weidner et al. (2013, red points), compared with the artificial star cluster distribution of 1000 models (black points) built using the RRS method with the calibrated expansion factor $P=6$ and the correction parameter $d=0.2$ (see Section 3 for details on the parameter $d$ ) and adding the errors of the same magnitude as for observations. The cyan long-dashed line is the theoretical relation derived by Kroupa et al. (2013) and the green solid line is a third order polynomial fit to the data of Weidner et al. (2013). The oblique black dashed line is a one-to-one relation between the mass of the most massive stars and the mass of their host clusters, $m_{\max }=M$, and the top black dashed line is the upper physical stellar mass limit, $150 M_{\odot}$.

cluster is conserved. To guarantee this, we shift the bins slightly, keeping their width $w_{\mathrm{OS}}$ constant, until the means of the bins coincide with the OS stars. For each bin, this consists in searching for the low-mass and high-mass limits of the bin, $a$ and $b$ (linked to $w_{\mathrm{OS}}$ by $w_{\mathrm{OS}}=b-a$ ), so that we respect the condition ${ }^{2}$ :

$$
m_{\mathrm{OS}}=\frac{m C D F(a, b)}{n C D F(a, b)},
$$

where $n C D F$ is the IMF number Cumulative Distribution Function (shown in the top panels of Fig. 1) and $m C D F$ is the mass Cumulative Distribution Function. We derive them in the following way.

We use the Kroupa (2001) IMF (shown in Fig. 1), defined as:

$$
\phi(m)=k \cdot C_{i} \cdot m^{-\alpha_{i}} \begin{cases}\alpha_{0}=0.3, & \text { if } m \in\left[m_{0}, m_{1}\right], \\ \alpha_{1}=1.3, & \text { if } m \in\left[m_{1}, m_{2}\right], \\ \alpha_{2}=2.3, & \text { if } m \in\left[m_{2}, m_{3}\right], \\ \alpha_{3}=2.3, & \text { if } m \in\left[m_{3}, m_{4}\right],\end{cases}
$$

\footnotetext{
${ }^{2}$ Following Kroupa (2002). For example, the mean mass of the whole range of mass, $[0.01,150] M_{\odot}$ of the Kroupa IMF, is $0.38 M_{\odot}$ (see Table 2 of Kroupa 2002).
} 
where $m_{0}=0.01 M_{\odot}, m_{1}=0.08 M_{\odot}, m_{2}=0.5 M_{\odot}, m_{3}=1 M_{\odot}, m_{4}=150 M_{\odot}$ (the upper physical stellar mass limit), and $k$ and $C_{i}$ are the normalization constants. The $n C D F$ and $m C D F$ are then defined as

$$
n C D F\left(m_{\mathrm{low}}, m\right)=\int_{m_{\mathrm{low}}}^{m} \phi\left(m^{\prime}\right) d m^{\prime},
$$

and

$$
m C D F\left(m_{\mathrm{low}}, m\right)=\int_{m_{\mathrm{low}}}^{m} m^{\prime} \phi\left(m^{\prime}\right) d m^{\prime} .
$$

The analytical expressions of $n C D F$ and $m C D F$ are

$$
n C D F\left(m_{\mathrm{low}}, m\right)=\sum_{j=0}^{i-1} \frac{k C_{j}}{1-\alpha_{j}}\left[m_{j+1}^{1-\alpha_{j}}-m_{j}^{1-\alpha_{j}}\right]+\frac{k C_{i}}{1-\alpha_{i}}\left[m^{1-\alpha_{i}}-m_{i}^{1-\alpha_{i}}\right]
$$

and

$$
m C D F\left(m_{\mathrm{low}}, m\right)=\sum_{j=0}^{i-1} \frac{k C_{j}}{2-\alpha_{j}}\left[m_{j+1}^{2-\alpha_{j}}-m_{j}^{2-\alpha_{j}}\right]+\frac{k C_{i}}{2-\alpha_{i}}\left[m^{2-\alpha_{i}}-m_{i}^{2-\alpha_{i}}\right]
$$

where $i$ is defined by $m \in\left[m_{i}, m_{i+1}\right]$ in Eq. ??.

Since $a$ and $b$ are related by $w_{\mathrm{OS}}=b-a$, Eq. ?? is solved for each bin. Thus, the mass generated in the bin is, on average, the same as the OS mass in that bin.

In step 3, the bins of all stars are expanded by multiplying their widths by the expansion factor, $w=P \cdot w_{\mathrm{O}}$. Then, each star is resampled in its individual bin. We could see the resampling as the application of the RS used in, e.g., Santos \& Frogel (1997), but restricted to the bins defined around each OS star position. The mass bins can be expanded for a transition toward RS $(P \rightarrow \infty)$ or narrowed for a transition toward OS $(P \rightarrow 0)$.

Fig. 3 shows the optimally sampled massive stars (gray vertical lines) of a star cluster with $\log _{10}\left(M / M_{\odot}\right)=4$, and the resampling bins for a few of the stars (the colored rectangles), for different values of the expansion factor $P$. When the bins are expanded too much, they can exceed the upper mass limit, $150 M_{\odot}$. To avoid this, we group the stars belonging to such bins and resample them within a common bin. In Fig. 3(c), the bin marked by a black solid line is a common bin for the 'red', 'blue', and 'green' stars of panels (a) and (b). The high-mass limit of the common bin is fixed to $b=150 M_{\odot}$, and the low-mass limit $a$ is found by requiring that the mean mass of the common bin equals the mean mass of the OS stars grouped into the common bin:

$$
\frac{m_{\mathrm{OS}_{1}}+m_{\mathrm{OS}_{2}}+\ldots+m_{\mathrm{OS}_{n}}}{n}=\frac{m C D F(a, 150)}{n C D F(a, 150)},
$$

where $n$ is the number of the stars grouped, e.g., $n=3$ in Fig. 3(c).

In a similar way, for low-mass stars close to their mass limit of $a=0.01 M_{\odot}$, a common bin is also defined by searching for the high-mass limit $b$.

When generating stars with the sampling algorithm described above and the bin widths defined as $w=P \cdot w_{\mathrm{OS}}$, we face a problem with the $m_{\max }$ vs. $M$ relation, displayed in the first column of the panels in Fig. 4. Indeed, for several values of the expansion factor (see cases $P=4,6)$, stars in low-mass clusters $\left(\log _{10}\left(m / M_{\odot}\right) \sim 2.5\right)$ almost reach the physical stellar mass limit, while it is not the case for more massive clusters. The reason is that, 


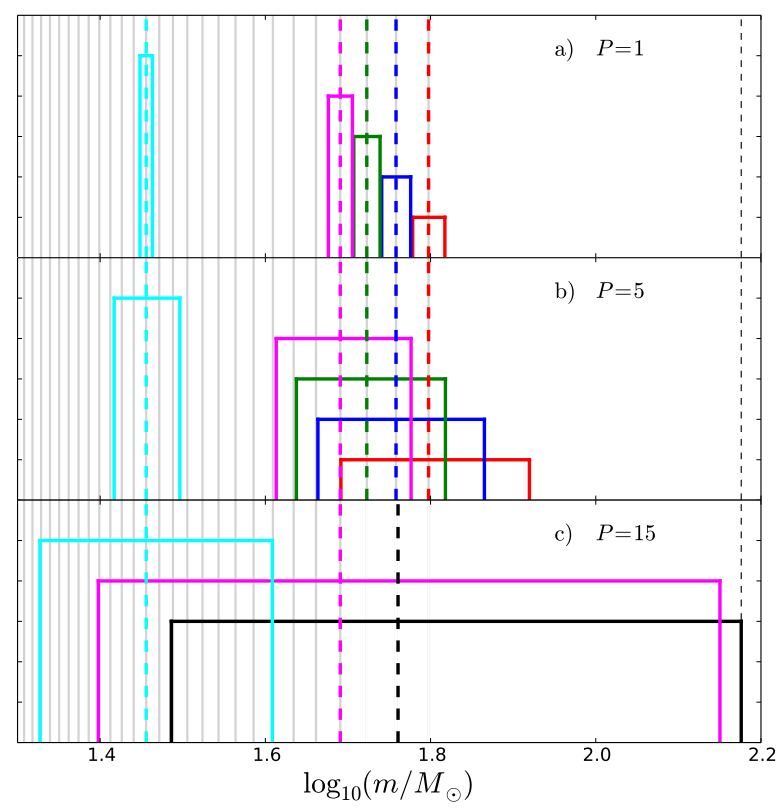

Fig. 3. Masses of the OS stars (gray vertical lines), with those for the $1^{\text {st }}, 2^{\text {nd }}, 3^{\text {rd }}, 4^{\text {th }}$, and $15^{\text {th }}$ most massive stars indicated by colored vertical dashed lines, respectively from right to left. The associated resampling bins are shown for several values of the expansion factors: $P=1$ (a), 5 (b), and 15 (c). In the case of a large enough expansion factor (c), the resampling bins of the three most massive stars (red, blue, and green) are grouped in a common bin (black solid line), in which they will be resampled. The mean mass of the bin, taken as the mean of the OS masses of the three stars, is shown by a thick black dashed line. The thin dashed line at the right marks the high-mass limit, $150 M_{\odot}$.

when we sample the stellar masses with OS, a low-mass cluster contains less stars than a massive cluster. Thus, the stars of the low-mass cluster are more distant in the mass space, creating around them larger bins. For the expansion factors $P=4$ and 6 , we see that this can create more massive stars in the low-mass clusters than in the more massive ones, what is unlikely to be true when considering the observational data from Weidner et al. (2013) (see red points in Fig. 2).

We applied a correction defined as a dependence of the bin width on the mass of the cluster:

$$
w(M)=P \cdot w_{\mathrm{OS}} \cdot\left(\frac{M}{10^{3} M_{\odot}}\right)^{d} .
$$

As it is demonstrated in Fig. 4, the choice of the power-law index, $d$, is guided by the fact that, for $d=0$ (see the first column of the panels), the effect is present, and for high power-law index ( $d=0.4$, see the last column of the panels), the $m_{\max }$ scatter present in massive clusters dominates too much, while it is reduced in low-mass clusters.

\section{CALIBRATION OF THE REDUCED RANDOM SAMPLING}

To calibrate the best value of the parameters $(P, d)$, we compare the observational data from Weidner et al. (2013) with the models built using the RRS method (see Fig. 2). We 


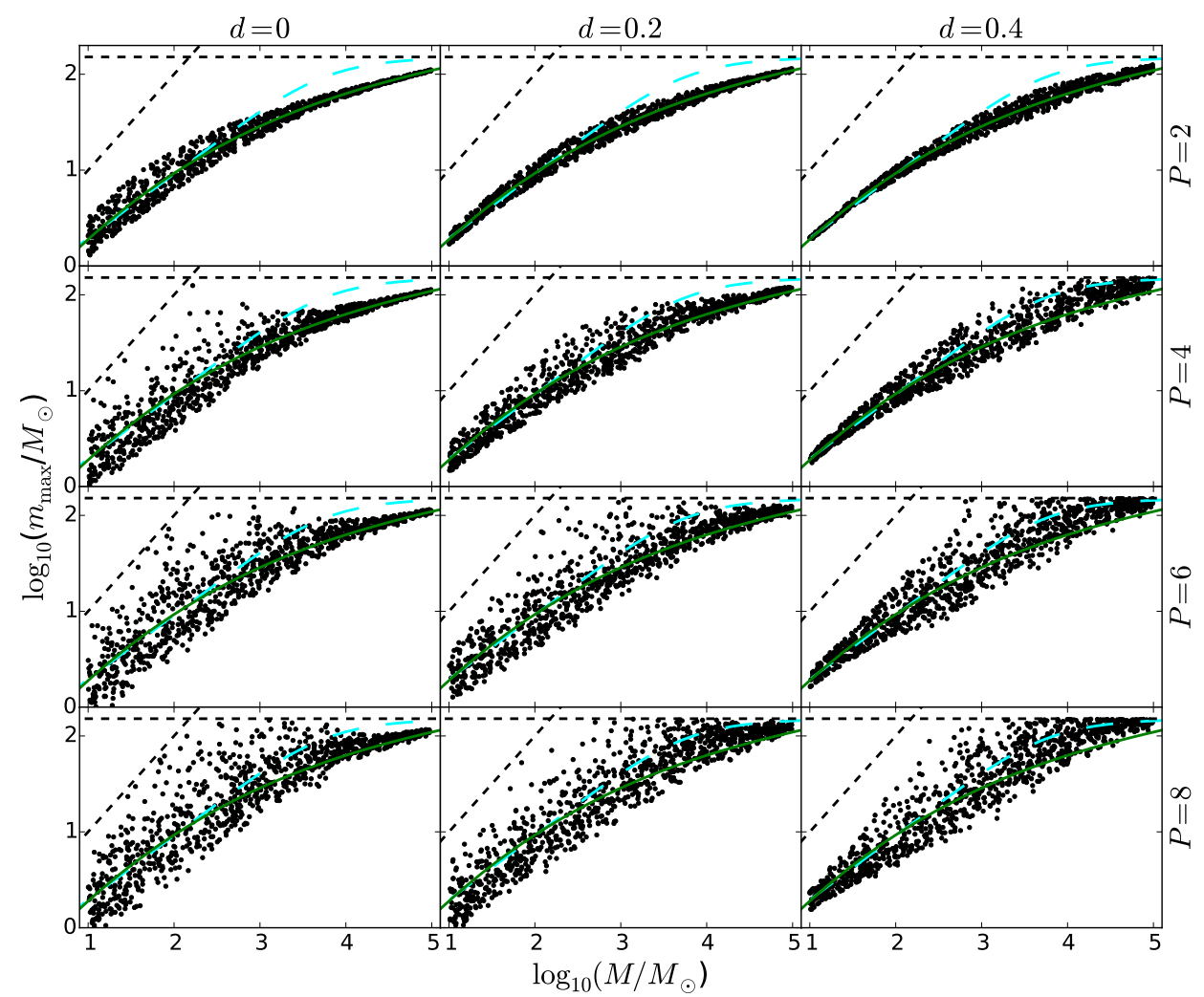

Fig. 4. Dependence of the stochasticity affecting the most massive stars of star clusters on the power-law index $d$ (indicated on the top) and on the expansion factor $P$ (indicated at the right of the figure). The first column of the panels presents the sampling when no correction is taken into account, $d=0$, while the central and right columns show the sampling generated with a growing correction. Each panel contains 1000 model clusters.

note that the error bars representing uncertainties in the mass of clusters and their most massive stars, provided by Weidner et al. (2013), are not statistical quantities but are just possible lower and higher limits. They derived the masses of the most massive stars using their spectral classes and assumed an error range as $1 / 2$ of the spectral subclass. For the masses of the clusters, they extrapolated them from the visible stars of the clusters using the IMF (Kroupa 2001). The upper mass limit was derived assuming that all stars could be unresolved binaries and the lower limit was set under the assumption that half of the stars were misidentified as cluster members.

We need a statistical description of the uncertainty to be introduced into the cluster models, and assume that it can be described by a Gaussian distribution with a value of sigma taken as $1 / 3$ of the Weidner et al. (2013) error bars. (Note that $99 \%$ of the measures fall within such uncertainty limits.)

We built several model distributions having different parameters $(P, d)$ and we added errors of the same value of sigma as defined above. To compare these distributions to the Weidner et al. (2013) data, we subdivide the model distributions and the observations in 10 groups according to the cluster mass in the range from $M=1$ to $M=4$. In each group $i$, we derive the likelihood function $l_{i}$ based on the comparison of the histogram 


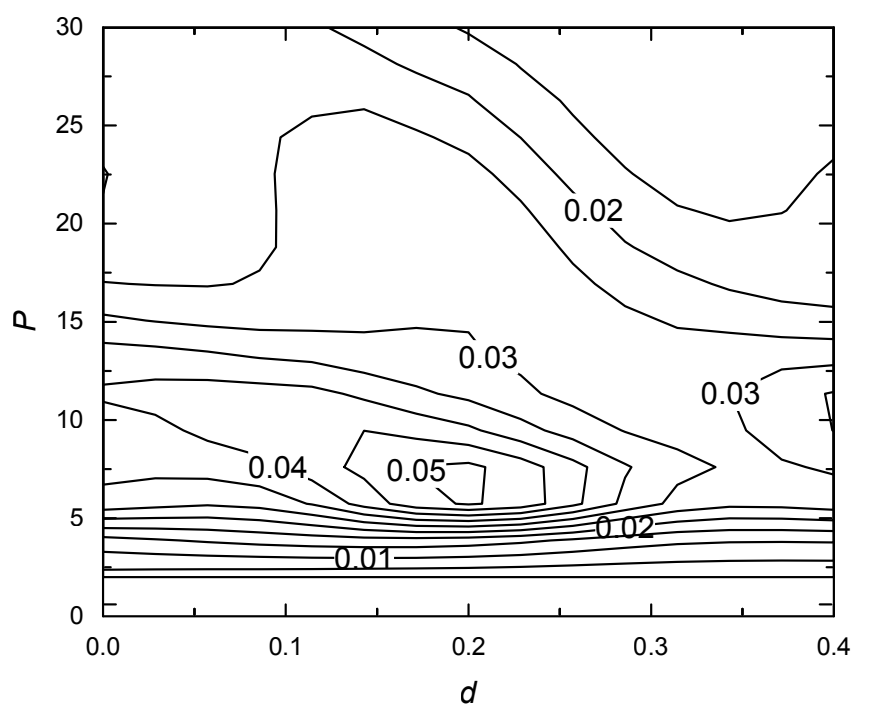

Fig. 5. Contour plot of the likelihood $L$ for the determination of the best combination of the parameters $(P, d)$, tested for different model distributions. The plots are normalized so that the sum of the likelihoods of all models is equal to 1 . The best combination is found at $P=6$ and $d=0.2$.

of the models minus the OS relation and the histogram of the observations minus the OS relation. Then, a total likelihood $L$ is derived as a product of the likelihoods $l_{i}$ of each group. The result is presented in Fig. 5, where the minimum is found at $P=6, d=0.2$.

We adopted these values of the parameters for the distribution shown in Fig. 2, which is compared to the observational data from Weidner et al. (2013). The few deviating points of observations do not influence significantly the selection of the best expansion factor, as neglecting these we got the same values of the parameters, $P=6$ and $d=0.2$. Note that Weidner et al. (2013) indicate that the errors provided by them do not take into account other sources of uncertainties, such as variable extinction, stellar variability, star loss due to gas expulsion and dynamical interactions. Hence, it is likely that the true expansion factor $P$ could be smaller than the one found here, which, therefore, should be regarded as an upper limit. More data and the refinement of the errors in the Weidner et al. (2013) sample would help to constrain the values of the parameters $(P, d)$ more accurately.

\section{CONCLUSIONS}

We present a new sampling method of stellar masses in clusters, the Reduced Random Sampling, which allows a continuous transition between the Optimal Sampling and the Random Sampling, already widely used in unresolved cluster studies. Using the catalog of young star clusters from Weidner et al. (2013), we constrained the parameters controlling the level of stochasticity in artificial clusters.

ACKNOWLEDGMENTS. This research was funded by a grant (No. MIP-074/2013) from the Research Council of Lithuania. 


\section{REFERENCES}

de Meulenaer P., Narbutis D., Mineikis T., Vansevičius V. 2013, A\&A, 550, A20

Deveikis V., Narbutis D., Stonkutė R. et al. 2008, Baltic Astronomy, 17, 351

Fouesneau M., Lançon A. 2010, A\&A, 521, A22

Kroupa P. 2001, MNRAS, 322, 231

Kroupa P. 2002, Science, 295, 82

Kroupa P., Weidner C. 2003, ApJ, 598, 1076

Kroupa P., Weidner C., Pflamm-Altenburg J. et al. 2013, in Planets, Stars and Stellar Systems, vol. 5, eds. T. D. Oswalt \& G. Gilmore, Springer, p. 115

Pflamm-Altenburg J., Kroupa P. 2006, MNRAS, 373, 295

Pflamm-Altenburg J., Weidner C., Kroupa P. 2007, ApJ, 671, 1550

Popescu B., Hanson M. M. 2013, ApJ, 780, 27

Santos J. F. C. Jr., Frogel J. A. 1997, ApJ, 479, 764

Weidner C., Kroupa P., Bonnell I. A. D. 2010, MNRAS, 401, 275

Weidner C., Kroupa P., Pflamm-Altenburg J. 2013, MNRAS, 434, 84 